\title{
The effect of initial lens diopter on myopia control by orthokeratology in Teenagers A retrospective Beijing Study
}

Jifeng Yu ( $\square$ jeffernyu@126.com )

Beijing Children's Hospital https://orcid.org/0000-0003-2365-4560

Wei Shi

Beijing Children's Hospital

Xue Liu

Beijing Children's Hospital

Si-Yuan Li

Beijing Children's Hospital

Li Li

Beijing Children's Hospital

Research article

Keywords: refractive error, myopia, orthokeratology

Posted Date: October 18th, 2019

DOl: https://doi.org/10.21203/rs.2.16212/v1

License: (1) (1) This work is licensed under a Creative Commons Attribution 4.0 International License.

Read Full License 


\section{Abstract}

Background To investigate the effect of initial refractive error on the control of myopia by orthokeratology.

Methods Retrospective study. Data were analyzed from 78 patients (156 eyes) who underwent orthokeratology in Beijing Children's Hospital from January 2014 to April 2018. The changes of axial length and diopter after wearing the lenses were measured. Patients were divided into six groups according to refractive error: $<-1.00 \mathrm{D},-1.00 \sim-2.00 \mathrm{D},-2.00 \mathrm{D} \sim-3.00 \mathrm{D},-3.00 \mathrm{D} \sim-4.00 \mathrm{D},-4.00 \mathrm{D} \sim-5.00 \mathrm{D}$ and $-5.00 \sim-6.00 \mathrm{D}$. A mixed-effect model was used to analyze the relevant data. At the same time, the interaction item between diopter and wearing time was analyzed, and the influence of gender was corrected.

Results Patients averaged 12.9 \pm 2.161 years old. Two years after wearing orthokeratology lenses, there was a statistically significant difference with the interaction item between refractive error and wearing time in each group. The estimated values were 0.0631 for the right eye and 0.053 for the left eye. Orthokeratology lenses controlled the progression of myopia well, but the reduction depended on the initial diopters. Binocular axial length examination didn't show statistically significant difference with the interaction item. Axial length increased with time, and the magnitude of increase did not differ among groups. However, the increase in the axial length of the male more than that of the female.

Conclusion Initial refractive errors have a significant impact on the control of myopia by orthokeratology. The less severe myopia patient demonstrated a more pronounced benefits after wearing orthokeratology lenses. Key words: refractive error; myopia; orthokeratology

\section{Background}

Myopia is the most common eye disease in the world. It is estimated that there are 1.406 billion people in the world who are myopic (22.9 percent of the population) and 163 million people have high myopia (2.7\% of the population). ${ }^{1}$ It is expected that the prevalence of myopia and high myopia will increase significantly around the globe, affecting nearly 5 billion people and 1 billion people by 2050, respectively. ${ }^{2}$ Myopia and high myopia affect 80 to 90 percent of young adults in many East Asian regions. ${ }^{3}$ The prevalence of myopia varies geographically. For example, in Australia ${ }^{4}$ and North America ${ }^{5}$ myopia affects $30 \%$ of the population, and in East Asia it affects as high as $85 \%$ of the population. ${ }^{6}$ Besides genetic factors, the increasing prevalence of myopia and high myopia may also be due to increasing pressure on education and less time for children to be outdoors, especially in East Asia.

How to effectively control the development of myopia is a worldwide problem. Recently, orthokeratology lenses have begun to gain popularity around the world. Orthokeratology lenses were first introduced by Wesley and Jessen in the 1950s and their design and materials have significantly improved. ${ }^{7}$ Orthokeratology can flatten the central cornea and delays axial elongation. ${ }^{8,9}$ Many literatures have 
confirmed the effectiveness of orthokeratology in controlling the progression of myopia and showed clinically acceptable safety. ${ }^{10,11}$ However, orthokeratology is still not widely used around the world. Most parents are worried about its safety, the skills that practitioners need to prescribe these lenses, the discomfort their children feel when wearing them overnight, and the costs. All these factors make parents hesitant to choose them which may lead to missing the best time for treatment and continued progression of myopia. Even with these questions, when to use orthokeratology and whether different refractive errors may lead to different outcomes have not been previously published.

This retrospective study aimed to investigate the effects of various factors of refractive errors, the relationship between refractive changes and different baseline factors, and to discuss the appropriate time to wear overnight OK lenses.

\section{Methods}

The study was a retrospective study of 78 school-aged children who visited private eye clinics between January 2014 and April 2019. Patients' inclusion and exclusion criteria in this study were strictly followed and were shown in Table 1.

All patients received detailed conventional ocular anteroposterior and tear film examinations before treatment and patients with acute and chronic ocular inflammation, dry eye, and other contraindications for wearing contact lenses were excluded. Patients with poor parental compliance, excessive anxiety and unrealistic expectations were excluded.

We divided all enrolled patients into six groups based on initial refractive error: $<-1.00 \mathrm{D},-1.00 \sim-2.00 \mathrm{D}$, $-2.00 \mathrm{D} \sim-3.00 \mathrm{D},-3.00 \mathrm{D} \sim-4.00 \mathrm{D},-4.00 \mathrm{D} \sim-5.00 \mathrm{D}$ and $-5.00 \sim-6.00 \mathrm{D}$.

Refractive examination of all patients included uncorrected visual acuity, subjective and objective refraction results. The compound tropicamide was used for dilated optometry before fitting. Auxiliary examinations also included non-contact intraocular pressure measurements, corneal thickness measurements, axial measurements (IOL master), corneal endothelium examinations, pupil diameter, and pentacam.

All patients in this study used night-time orthokeratology lenses and worn 8-10 hours at night. Lenses were fitted according to the patient's corneal curvature and other parameters. The appropriate lenses were selected to try on and were fluorescein stained after $30 \mathrm{~min}$. The lenses were observed under a slit lamp to determine the adaptation of the lenses, the lens' base arc, and then the right orthokeratology lenses were chosen. Patients' parents were instructed on how to wear and maintain the lenses and were asked to strictly observe the wearing time.

After the first night of wear, all patients were followed up on the next day, the next week and the next month, and then every three months thereafter. If there is abnormality in the eye, patients were asked to visit the doctor at any time. At the time of follow-up, the status of the anterior segment of the eye was 
examined using a slit lamp, which, combined with the optometry evaluation and corneal topography, determined the fitting of the lenses. Axial length was measured 1 year and 2 years after wearing lenses. The measurement time of all follow-ups was scheduled to be completed within 2 hours after the lenses were removed in the morning.

Data was processed via GraphPad Prism5 software (GraphPad Software, Inc., La Jolla, CA, USA). The statistical analysis used a mixed-effects model to analyze the interactive items of lens diopter and wearing time while correcting the influence of gender.

The study was conducted at the Department of Ophthalmology, Beijing Children's Hospital, Capital Medical University and Beijing National Children's Health Center. The research program was approved by the Ethics Committee of Beijing Children's Hospital. All patients and the parents of the control group agreed to the study procedure, and all the experiments in the project met the principles of the Helsinki Declaration.

\section{Results}

\section{- STUDY POPULATION}

The details of the 78 subjects are shown in Table 2.

- Changes in diopters after wearing orthokeratology lenses in different groups

After 2 years of wearing the orthokeratology lenses, diopter and axial length changes of the patients in each group were evaluated separately for each eye. The right eye's diopter and wearing time interaction item (group*time) was statistically significant (Table 3), and the estimated value was positive (0.0631). The results of the left eye's were exactly the same as those of the right eye (Table 4) and the estimate is 0.053 .

In the analysis of binocular axial length of all patients, there was no statistical significance with the interaction items, ie, in different diopter groups (whether $-1.00 \mathrm{D}$ or $-6.00 \mathrm{D}$ ), the binocular axial length increased with the increase of time, and the increase magnitude among groups showed no difference.

The analysis of the right eye's axis showed that there was no statistically significant difference with the interaction item between diopter and wearing time $(P=0.8469)$, so only the main effects of diopter and wearing time were analyzed (Table 5).

The results showed that with each 1.00D increase in diopter (group), axial length of the right eye increased by $0.266 \mathrm{~mm}$; with each additional year, the axial length of the right eye increased by $0.1512 \mathrm{~mm}$; the increase in male was $0.4014 \mathrm{~mm}$ more than that in female.

The analysis of the left eye's axis showed that there was no statistically significant difference with the interaction item between diopter and wearing time $(P=0.7606)$, so only the main effects of diopter and 
wearing time were analyzed (Table 6).

The results showed that with each 1.00D increase in diopter (group), axial length increased by $0.2349 \mathrm{~mm}$; with each additional year, axial length increased by $0.1386 \mathrm{~mm}$; the increase in male was $0.3885 \mathrm{~mm}$ more than that in female.

\section{Discussion}

As the incidence of myopia continues to rise, the age of onset is getting younger and the proportion of high myopia is growing. How to control the development of myopia and possible harm is a problem that ophthalmologists and parents are very concerned about. ${ }^{2}$ Diopter and axial length are the two main examination indicators to evaluate the development of myopia. At present, various methods to control myopia mainly focused on these two aspects. Huang's meta-analysis showed that a series of interventions can significantly reduce the progression of myopia compared with single-lens or placebo. The most effective interventions are pharmacological, such as muscarinic antagonists including atropine and pirenzepine. Orthokeratology and peripheral defocusing corrective contact lenses are moderately effective in controlling the progression of myopia. ${ }^{12}$ Unfortunately, drugs such as low concentrations of atropine eye drops have not yet been marketed in mainland China. As a result, children with myopia are still unable to use them. In addition, long-term use of atropine may cause side effects and long-term efficacy is still controversial. ${ }^{13,14}$ Orthokeratology in China is still the main method of controlling myopia. Most of the previous studies were related to the study of the safety and effectiveness of long-term use of orthokeratology lenses. Because the users of orthokeratology lenses are children, parents' concern about safety, the degree of compliance when children try to wear these lenses, and relatively expensive prices have led to many children unable to use orthokeratology lenses to control myopia. There are currently fewer studies to address the issue of whether the use of orthokeratology lenses at different diopters will affect the outcomes. In view of this, we systematically reviewed the related data of children who used orthokeratology lenses in our hospital between January 2014 and April 2018 and grouped them according to their diopters at the time of them first wearing lenses. We compare the efficacy of different groups after wearing lenses and then discussed the impact of orthokeratology lenses on children with different diopters. All children enrolled in the team were able to adhere well and had regular follow-ups. Both parents and children showed good compliance. We used a standard lens design and apply it to children with low to moderate myopia. These patients who don't have astigmatism or have low astigmatism do not require the change of the lens design. All patients in this group achieved satisfactory corneal reshaping results.

Our study found that after 2 years of wearing orthokeratology lenses, there were statistically significant differences with the interaction item between diopters and wearing times for each group, and the estimated values were positive (OD: 0.0631D, OS 0.053D). This showed that the increase of binocular diopters in different groups would be reduced with the increase of wearing time, and orthokeratology lenses controlled the progression of myopia well. However, among groups, the diopter increase reduction was different. For each 1.0D increase of diopters, the right eye diopter increase will go up by more than 
$0.0631 \mathrm{D}$, while that of the left eye will rise by $0.053 \mathrm{D}$. In a retrospective study conducted by Lee in 2017 , a long period of follow-ups (up to 12 years) showed that overnight OK lenses can significantly affect the progression of refractive errors in children with myopia. The increase of diopters of the OK group was approximately 0.2 to 0.3 per year, while that of the glasses-wearing group was approximately 0.4 to 0.5 per year. Their results showed that orthokeratology can control myopia for up to 8 years. ${ }^{15,16}$ However, other studies reported that the time-dependence of using OK lenses significantly reduced the efficacy of their myopic controls. It was confirmed that even without OK lenses, the diopters of myopic children would drop, which is a natural process. ${ }^{11,17-19}$ This means that as age increases, the difference between the OK group and the control group gradually decreases. However, our study suggests that there are differences in the control of diopters by OK lens. The higher the diopter, the poorer the efficacy. As age increases, children's diopters are also rising, indicating that age and diopter may be factors in the decline of OK lenses' efficacy.

In our study, the results of the axial length comparison showed that there was no statistical significance with the interaction item in all groups, i.e., in all groups, both eyes' axis length increased over time and the magnitude of increase in each group was not different. This shows that although orthokeratology lenses can significantly control the elongation of the axial length, ${ }^{20-23}$ regardless of the diopter of the orthokeratology lens, its control of the axial length elongation does not differ significantly. It is interesting that the magnitude of elongation of the binocular axial length was related to gender. Our results showed that with each additional year of wearing lenses, the axial length of the right eye increased by $0.1512 \mathrm{~mm}$; male had an additional increase of $0.4014 \mathrm{~mm}$ over female. The axial length of the left eye increased by $0.1386 \mathrm{~mm}$; males had an additional increase of $0.3885 \mathrm{~mm}$ over female. In other words, in terms of controlling the increase of the ocular axis by orthokeratology lens, there was a difference between genders and it was more effective for female. This may be related to stronger compliance with adolescent women compared to men, which led to better outcomes. ${ }^{15,22}$

\section{Conclusions}

In summary, we performed a statistical analysis of children wearing orthokeratology lenses that had been followed for 2 years. The study found that orthokeratology lenses should be worn as soon as possible after the diagnosis of myopia as early wearing of orthokeratology lenses can better control the progression of myopia. The group of children with less than 100 degrees of diopters showed significantly better results than the high myopia groups. There were no significant differences between diopter and axial length increase, but for female children, wearing orthokeratology lenses when diopters were low proved to be more efficacious in controlling axial length elongation than for male children.

The limitations of our study are the small number of subjects, the short follow-up time and the lack of multicenter controlled studies. In addition, the average age of children in the group was $12.9 \pm 2.16$ while the current age of myopia diagnosis is getting younger. Whether or not children in 7-10 years of age still have such a pattern requires further discussion. As a next step, we will collect more clinical cases and conduct in-depth systematic research on more clinical centers inside and outside the country. 


\section{Declarations}

\section{Abbreviations}

OK: orthokeratology; D: diopter.

\section{Acknowledgements}

Not applicable.

\section{Authors' contributions}

Conceived and designed the study: JFY, WS, and XL; Acquisition of data: XL and SYL; Analysis and interpretation of data: JFY, WS, and XL; Drafting the manuscript: JFY, WS, and XL; Revising the manuscript critically for important intellectual content: LL. All authors read and approved the final manuscript.

\section{Funding}

Ophthalmic data collection, statistical analysis and publication charges in the study were supported by BeiJing Talents Fund囚2015000021469G208囚

\section{Availability of data and materials}

The data used to support the findings of this study are available from the corresponding author upon request.

\section{Ethics approval and consent to participate}

This study was performed in accordance with the Declaration of Helsinki and was approved by the Ethics Committee of Beijing Children's Hospital, Beijing, China. Informed consent was obtained from the parents of all the subjects after the aims and nature of the study were explained to them. Written informed consents were obtained from the parents of all the subjects participating in the investigation.

\section{Consent for publication}

Not applicable.

\section{Competing interests}


The authors declare that they have no competing interests.

\section{Author details}

Department of Ophthalmology, Beijing Children's Hospital, Capital Medical University, National Center for Children's Health, Address: 56 South Lishi Road, Beijing 100045, China.

\section{References}

1. Chua J, Wong TY. Myopia-The Silent Epidemic That Should Not Be Ignored. JAMA Ophthalmol. 2016; 12:1363-1364.

2. Holden BA, Fricke TR, Wilson DA, et al. Global Prevalence of Myopia and High Myopia and Temporal Trends from 2000 through 2050. Ophthalmology. 2016; 5:1036-42.

3. Ding BY, Shih YF, Lin LLK, et al. Myopia among schoolchildren in East Asia and Singapore. Surv Ophthalmol. 2017; 5:677-697.

4. Wensor M, McCarty CA, Taylor HR. Prevalence and risk factors of myopia in Victoria, Australia. Arch Ophthalmol. 1999; 5:658-63.

5. Vitale S, Ellwein L, Cotch MF, et al. Prevalence of refractive error in the United States, 1999-2004. Arch Ophthalmol. 2008; 8:1111-9.

6. Wang TJ, Chiang TH, Wang TH, et al. Changes of the ocular refraction among freshmen in National Taiwan University between 1988 and 2005. Eye (Lond). 2009; 5:1168-9.

7. Swarbrick HA. Orthokeratology review and update. Clin Exp Optom. 2006; 3:124-43.

8. Queiros A, Gonzalez-Meijome JM, Jorge J, et al. Peripheral refraction in myopic patients after orthokeratology. Optom Vis Sci. 2010; 5:323-9.

9. Sankaridurg P, Holden B, Smith E, 3rd, et al. Decrease in rate of myopia progression with a contact lens designed to reduce relative peripheral hyperopia: one-year results. Invest Ophthalmol Vis Sci. 2011; 13:9362-7.

10. Cho P, Cheung SW, Edwards M. The longitudinal orthokeratology research in children (LORIC) in Hong Kong: a pilot study on refractive changes and myopic control. Curr Eye Res. 2005; 1:71-80.

11. Hiraoka T, Kakita T, Okamoto F, et al. Long-term effect of overnight orthokeratology on axial length elongation in childhood myopia: a 5-year follow-up study. Invest Ophthalmol Vis Sci. 2012; 7:3913-9.

12. Huang J, Wen D, Wang Q, et al. Efficacy Comparison of 16 Interventions for Myopia Control in Children: A Network Meta-analysis. Ophthalmology. 2016; 4:697-708.

13. Chia A, Chua WH, Wen L, et al. Atropine for the treatment of childhood myopia: changes after stopping atropine $0.01 \%, 0.1 \%$ and $0.5 \%$. Am J Ophthalmol. 2014; 2:451-457 e1.

14. Chia A, Lu QS, Tan D. Five-Year Clinical Trial on Atropine for the Treatment of Myopia 2: Myopia Control with Atropine 0.01\% Eyedrops. Ophthalmology. 2016; 2:391-9. 
15. Lee YC, Wang JH, Chiu CJ. Effect of Orthokeratology on myopia progression: twelve-year results of a retrospective cohort study. BMC Ophthalmol. 2017; 1:243.

16. Cheung SW, Cho P, Chui WS, et al. Refractive error and visual acuity changes in orthokeratology patients. Optom Vis Sci. 2007; 5:410-6.

17. Kakita T, Hiraoka T, Oshika T. Influence of overnight orthokeratology on axial elongation in childhood myopia. Invest Ophthalmol Vis Sci. 2011; 5:2170-4.

18. Fan Q, Teo YY, Saw SM. Application of advanced statistics in ophthalmology. Invest Ophthalmol Vis Sci. 2011; 9:6059-65.

19. Donovan L, Sankaridurg P, Ho A, et al. Myopia progression rates in urban children wearing singlevision spectacles. Optom Vis Sci. 2012; 1:27-32.

20. Chen Z, Zhou J, Qu X, et al. Effects of orthokeratology on axial length growth in myopic anisometropes. Cont Lens Anterior Eye. 2018; 3:263-266.

21. Cho P, Cheung SW. Protective Role of Orthokeratology in Reducing Risk of Rapid Axial Elongation: A Reanalysis of Data From the ROMIO and TO-SEE Studies. Invest Ophthalmol Vis Sci. 2017; 3:14111416.

22. Leo SW, Scientific Bureau of World Society of Paediatric O, Strabismus. Current approaches to myopia control. Curr Opin Ophthalmol. 2017; 3:267-275.

23. Li X, Friedman IB, Medow NB, et al. Update on Orthokeratology in Managing Progressive Myopia in Children: Efficacy, Mechanisms, and Concerns. J Pediatr Ophthalmol Strabismus. 2017; 3:142-148.

\section{Tables}

Table 1: Patient inclusion and exclusion criteria

\begin{tabular}{|c|c|}
\hline Inclusion Criteria & Exclusion Criteria \\
\hline Between 7 to 18 years of age & Strabismus at distance or near \\
\hline $\begin{array}{l}\text { Myopia: between }-0.50 \mathrm{D} \text { and }-6.00 \mathrm{D} \text { in at } \\
\text { least } 1 \text { eye }\end{array}$ & $\begin{array}{l}\text { Previous experience in contact lens wear or } \\
\text { various myopia control treatment (e.g., } \\
\text { refractive therapy or progressive spectacles) }\end{array}$ \\
\hline $\begin{array}{c}\text { Astigmatism: }<1.50 \mathrm{D} ; \text { with-the-rule } \\
\text { astigmatism (axes } 180 \pm 30) \leq 1.25 \mathrm{D} ; \\
\text { astigmatism of other axes } \leq 0.50 \mathrm{D} \text { in both } \\
\text { eyes }\end{array}$ & $\begin{array}{l}\text { Past eye disease history, trauma, tumor, } \\
\text { corneal dystrophy, dry eye, etc. }\end{array}$ \\
\hline $\begin{array}{l}\text { distant best-corrected visual acuity (BCVA) } \\
\text { better than } 0 \text { log minimum angle of } \\
\text { resolution (logMAR) units (20/20) } \\
\text { follow-up period greater than } 2 \text { years }\end{array}$ & $\begin{array}{l}\text { Poor compliance, parental anxiety, cannot } \\
\text { strictly comply with the corresponding nursing } \\
\text { measures and review requirements }\end{array}$ \\
\hline
\end{tabular}


Table 2: Basic conditions of the enrolled patients

\begin{tabular}{|c|c|c|c|c|c|c|}
\hline $\begin{array}{l}\text { Diopters } \\
\text { groups }\end{array}$ & Age & $\begin{array}{c}\text { Gender } \\
\text { (male/female) }\end{array}$ & $\begin{array}{l}\text { Diopter } \\
\text { Right }\end{array}$ & $\begin{array}{l}\text { Diopter } \\
\text { Left }\end{array}$ & $\begin{array}{l}\text { Axial } \\
\text { length } \\
\text { Right }\end{array}$ & $\begin{array}{c}\text { Axial } \\
\text { length left }\end{array}$ \\
\hline$<-1.00 \mathrm{D}$ & $11.7 \pm 2.21$ & $4 / 6$ & $-0.83 \pm 0.31$ & $-0.73 \pm 0.27$ & $23.85 \pm 0.47$ & $23.69 \pm 0.42$ \\
\hline $\begin{array}{l}-1.00 \mathrm{D} \\
\sim-2.00 \mathrm{D}\end{array}$ & $12.45 \pm 1.81$ & $9 / 8$ & $-1.54 \pm 0.31$ & $-1.56 \pm 0.37$ & $23.86 \pm 0.69$ & $23.91 \pm 0.69$ \\
\hline $\begin{array}{l}-2.00 \mathrm{D} \\
\sim-3.00 \mathrm{D}\end{array}$ & $12.5 \pm 1.90$ & $4 / 8$ & $-2.35 \pm 0.29$ & $-2.42 \pm 0.62$ & $23.92 \pm 0.54$ & $23.86 \pm 0.55$ \\
\hline $\begin{array}{l}-3.00 \mathrm{D} \\
\sim-4.00 \mathrm{D}\end{array}$ & $13.75 \pm 2.17$ & $10 / 10$ & $-3.36 \pm 0.26$ & $-3.37 \pm 0.58$ & $24.85 \pm 0.63$ & $24.83 \pm 0.73$ \\
\hline $\begin{array}{l}-4.00 \mathrm{D} \sim \\
-5.00 \mathrm{D}\end{array}$ & $13.0 \pm 2.41$ & $6 / 6$ & $-4.19 \pm 0.26$ & $-4.11 \pm 0.58$ & $24.63 \pm 0.85$ & $24.3 \pm 0.77$ \\
\hline $\begin{array}{l}-5.00 \mathrm{D} \sim \\
-6.00 \mathrm{D}\end{array}$ & $13.29 \pm 2.05$ & $4 / 3$ & $-5.21 \pm 0.17$ & $-5.20 \pm 0.27$ & $5.1 \pm 0.82$ & $24.98 \pm 0.66$ \\
\hline Total & $12.9 \pm 2.161$ & $37 / 41$ & $-2.82 \pm 0.35$ & $-2.78 \pm 0.37$ & $24.35 \pm 0.81$ & $24.26 \pm 0.79$ \\
\hline
\end{tabular}

Table 3 Diopter and wearing time interaction item (group*time) in right eye

\begin{tabular}{|c|c|c|c|c|c|}
\hline Effects & Estimates & Standard deviation Degree of & Freedom & T Value & $\operatorname{Pr}>|t|$ \\
\hline group & -0.9455 & 0.04277 & 147 & -22.11 & $<.0001$ \\
\hline time & -0.3743 & 0.04848 & 147 & -7.72 & $<.0001$ \\
\hline group *time & 0.06306 & 0.01737 & 147 & 3.63 & 0.0004 \\
\hline gender & -0.1254 & 0.07686 & 147 & -1.63 & 0.1048 \\
\hline
\end{tabular}

Table 4 Diopter and wearing time interaction item (group*time) in left eye

\begin{tabular}{lrrrrr}
\hline Effects & Estimates & Standard Deviation & Degree of Freedom & T Value & Pr $>|\mathrm{t}|$ \\
\hline group & -0.8460 & 0.05388 & 146 & -15.70 & $<.0001$ \\
time & -0.3141 & 0.04846 & 146 & -6.48 & $<.0001$ \\
group*time & 0.05285 & 0.01736 & 146 & 3.04 & 0.0028 \\
gender & -0.1531 & 0.1270 & 146 & -1.21 & 0.2298 \\
\hline
\end{tabular}


Table 5 Axial length and wearing time interaction item (group*time) in right eye

\begin{tabular}{lrrrrr}
\hline Effects & Estimates & Standard Deviation & Degree of Freedom & T Value & Pr $>|t|$ \\
\hline group & 0.2660 & 0.04833 & 148 & $5.51<.0001$ \\
time & 0.1512 & 0.02043 & 148 & $7.40<.0001$ \\
gender & 0.4014 & 0.1490 & 148 & 2.69 & 0.0079 \\
\hline
\end{tabular}

Table 6 Axial length and wearing time interaction item (group*time) in left eye

\begin{tabular}{|c|c|c|c|c|c|}
\hline Effects & Estimates & Standard deviation & Degree of Freedom & T Value & $\operatorname{Pr}>|t|$ \\
\hline group & 0.2349 & 0.04936 & 147 & 4.76 & $<.0001$ \\
\hline time & 0.1386 & 0.02302 & 147 & 6.02 & $<.0001$ \\
\hline gender & 0.3835 & 0.1522 & 147 & 2.52 & 0.0128 \\
\hline
\end{tabular}

